\title{
The Effects of Menopausal Hormone Therapy on Serum Level of C-reactive Protein in Postmenopausal Korean Women
}

\author{
Whan Shin, Sung Eun Kim, Jee-Yeon Lee, Jong-Wook Seo, Hye Sun Hyun, Ji Hyun Suh, DooSeok Choi, \\ Byung-Koo Yoon \\ Department of Obstetrics, Gynecology, and Women's Health, Samsung Medical Center, Sungkyunkwan University School of Medicine, Seoul,
} Korea

Objectives: Inflammation is a major mechanism underlying coronary heart disease (CHD) and C-reactive protein (CRP) is a marker of inflammation. When administered soon after menopause, menopausal hormone therapy (MHT) prevents CHD. This study was conducted to examine the impact of estrogen by administration route on CRP in postmenopausal Korean women using micronized progesterone (MP4) for endometrial protection.

Methods: This retrospective cohort study included 129 healthy women without CHD risk factors. Eighty-nine women took oral estrogen (conjugated equine estrogen, $0.625 \mathrm{mg} /$ day or equivalent), and 40 women applied a $1.5-\mathrm{mg} /$ day $0.1 \%$ percutaneous estradiol gel. MP4 was added in 82 women with an intact uterus. The CRP level was measured at baseline and three and six months after initiation of MHT.

Results: The baseline characteristics were comparable between the MHT groups except current age and age at menopause. After controlling for age, menopausal age, body mass index, and basal CRP, no significant change in CRP was observed in the oral estrogen group $(n=29)$. Follow-up CRP levels were also similar to the baseline in the percutaneous estrogen group $(n=$ 18). However, three-month CRP was significantly lower than six-month CRP, and there was a significant time trend within the percutaneous estrogen group. However, the group difference did not reach statistical significance. CRP also did not differ by addition of MP4 in either group.

Conclusions: In postmenopausal Korean women, no change in CRP was observed with oral estrogen, while percutaneous estrogen might decrease CRP. The estrogenic impacts were not influenced by adding MP4. (J Menopausal Med 2019;25:49-54)

Key Words: C-reactive protein · Hormone replacement therapy · Progesterone · Postmenopause · Drug administration routes

\section{Introduction}

Inflammation is one of the main pathophysiology of coronary heart disease (CHD), ${ }^{1}$ which leads to heightened plaque instability and a propensity for thrombosis. ${ }^{2}$ C-reactive protein (CRP) is an inflammatory marker, which is produced by hepatocytes in response to inflammatory cytokines including interleukin-6 (IL-6) and tumor necrosis factor $\alpha(\mathrm{TNF}-\alpha){ }^{3.6}$
Elevated blood level of CRP is associated with CHD events, and $\mathrm{CRP}$ is considered as a risk marker for $\mathrm{CHD}{ }^{7}$

CHD is a major cause of mobility and mortality in women, ${ }^{8,9}$ and the incidence of CHD increases after menopause. ${ }^{8,10,11}$ Previous observational studies have suggested that there are long-term benefits of menopausal hormone therapy (MHT) for reducing the risk ${ }^{12,13}$ or mortality ${ }^{1416}$ as sociated with CHD. Recently, a timing hypothesis has been

Received: September 30, 2018 Revised: November 20, 2018 Accepted: December 14, 2018

Address for Correspondence: Byung-Koo Yoon, Department of Obstetrics, Gynecology, and Women's Health, Samsung Medical Center, Sungkyunkwan University School of Medicine, 81 Irwon-ro, Gangnam-gu, Seoul 06351, Korea

Tel: +82-2-3410-3519, Fax: +82-2-3410-0630, E-mail: bkyoon@skku.edu

ORCID: https://orcid.org/0000-0002-1326-6102

Copyright (c) 2019 by The Korean Society of Menopause

(c) This is an Open Access article distributed under the terms of the Creative Commons Attribution Non-Commercial License (http://creativecommons.org/licenses/by-nc/4.0/). 
raised. Randomized controlled trials have demonstrated that women receiving MHT had significantly reduced risk of both $\mathrm{CHD}$ and total mortality in early menopause but not in late menopause. ${ }^{17}$

Estrogen deficiency increases inflammation in the vessel walls. ${ }^{18}$ MHT is reported to decrease blood inflammation markers such as cell adhesion molecules and chemokines. ${ }^{19,20}$ However, oral estrogen therapy increases the plasma concentrations of $\mathrm{CRP},{ }^{21}$ suggesting the increase in CRP is a metabolic response.

In the Women's Health Initiative Study, an increased risk of $\mathrm{CHD}$ was associated with conjugated equine estrogen (CEE) plus medroxyprogesterone acetate (MPA) compared with CEE alone. ${ }^{22}$ While MPA may negate the beneficial effects of estrogen on blood vessels, micronized progesterone (MP4) does not counteract estrogen-mediated effects on blood vessels. ${ }^{23}$

Based on these findings, we investigated the changes in CRP level according to route of estrogen administration and also the effect of MP4 added to estrogen in postmenopausal Korean women.

\section{Materials and Methods}

\section{Participants}

We enrolled 129 healthy postmenopausal women who visited Samsung Medical Center Menopause Clinic in Seoul, Korea between January 2001 and December 2013. Menopause was defined when women had been amenorrheic for at least 12 months or had a serum follicle stimulating hormone level $>40 \mathrm{IU} / \mathrm{L}$. Patients with a history of $\mathrm{CHD}$ or its risk factors, such as hypertension, diabetes mellitus, dyslipidemia, and smoking, were excluded. Women with chronic inflammatory disease, including rheumatologic disease, steroid use, malignant diseases, or contraindications for MHT, were also excluded.

\section{Study design}

In this retrospective cohort study, the participants were divided into oral and percutaneous MHT groups according to route of estrogen administration. As for estrogen, CEE 0.625 mg/day (Wyeth Korea, Seoul, Korea) or equivalent and per- cutaneous $0.1 \%$ estradiol gel $1.5 \mathrm{mg} /$ day (Samil Pharma Co., Seoul, Korea) were given to the oral and parenteral MHT groups, respectively. MP4 (Hanhwa Pharma Co., Seoul, Korea) was added in women with an intact uterus either sequentially (200 mg/day for 12 days, monthly) or continuously (100 mg/day). All patients were followed up for at least six months, and there was no regimen change during the study period. The study protocol was approved by the Institutional Review Board of Samsung Medical Center (IRB No. 2015-10163).

\section{CRP measurement}

High-sensitivity CRP assay was used because it more precisely assesses low-level proteins. ${ }^{24}$ Serum CRP level was measured before MHT initiation and again after three and six months of MHT by immunoturbidimetry assay (Roche Modulator P analyser; Roche, Seoul, Korea).

\section{Statistical analysis}

Data were analysed with a standard statistical package (SAS version 9.4; SAS Institute, Cary, NC, USA). Data are expressed as mean \pm standard deviation or number. Baseline characteristics were assessed using the $t$ test, Wilcoxon rank sum test, chi-square test, or Fisher's exact test according to the type of data. Group differences at each time point and for time trends adjusting for covariates were analysed using generalized estimating equations. A multiple linear regression analysis was employed to evaluate the interaction effect of MP4 on serum CRP level adjusting for covariates. Because the distribution of CRP was skewed, a logarithmic transformation was used. All $P$ values were two-sided, and values $<0.05$ were considered statistically significant,

\section{Results}

The baseline characteristics of the study participants are shown in Table 1. All variables except current age and menopausal age were comparable between the two groups. The distributions of participants by estrogen route of administration and addition of MP4 are also included in Table 1.

After controlling for age, menopausal age, body mass index, and basal CRP, the effects of estrogen alone on CRP 
Table 1. Baseline characteristics of the study participants according to route of estrogen administration

\begin{tabular}{|c|c|c|c|c|}
\hline \multirow{2}{*}{ Characteristic } & \multicolumn{2}{|c|}{ Oral MHT $(n=89)$} & \multicolumn{2}{|c|}{ Percutaneous MHT $(n=40)$} \\
\hline & Estrogen only $(n=29)$ & Estrogen + MP4 $(n=60)$ & Estrogen only $(n=18)$ & Estrogen + MP4 $(n=22)$ \\
\hline Age (y) & $53.3 \pm 5.4^{*}$ & $55.7 \pm 5.0^{*}$ & $52.5 \pm 4.1$ & $54.0 \pm 4.7$ \\
\hline $\operatorname{BMl}\left(\mathrm{kg} / \mathrm{m}^{2}\right)$ & $22.0 \pm 2.3$ & $22.5 \pm 2.3$ & $22.6 \pm 2.6$ & $21.8 \pm 1.8$ \\
\hline Age at menarche $(y)$ & $15.0 \pm 1.4$ & $15.3 \pm 1.6$ & $15.6 \pm 1.8$ & $15.6 \pm 1.7$ \\
\hline Menopausal age (y) & $42.8 \pm 7.1^{+, \neq}$ & $50.7 \pm 3.5^{+}$ & $42.1 \pm 5.0^{\ddagger \S}$ & $49.5 \pm 3.4^{\S}$ \\
\hline \multicolumn{5}{|l|}{ Type of menopause } \\
\hline Natural & 0 & 59 & 1 & 22 \\
\hline Surgical & 29 & 1 & 17 & 0 \\
\hline Systolic BP (mmHg) & $122.2 \pm 17.1$ & $115.6 \pm 14.2$ & $117.2 \pm 14.2$ & $119.8 \pm 15.5$ \\
\hline Diastolic BP $(\mathrm{mmHg})$ & $74.3 \pm 9.9$ & $71.8 \pm 10.5$ & $68.0 \pm 8.3$ & $73.4 \pm 13.6$ \\
\hline Glucose (mg/dL) & $91.1 \pm 6.9$ & $94.2 \pm 8.9$ & $92.9 \pm 8.2$ & $97.3 \pm 10.2$ \\
\hline Total-C (mg/dL) & $197.7 \pm 28.9$ & $203.2 \pm 32.2$ & $217.1 \pm 37.1$ & $199.2 \pm 37.6$ \\
\hline $\mathrm{TG}(\mathrm{mg} / \mathrm{dL})$ & $99.5 \pm 38.8$ & $94.8 \pm 41.8$ & $115.3 \pm 57.8$ & $95.0 \pm 48.1$ \\
\hline $\mathrm{HDL}-\mathrm{C}(\mathrm{mg} / \mathrm{dL})$ & $63.6 \pm 12.8$ & $63.7 \pm 15.9$ & $63.2 \pm 13.1$ & $64.6 \pm 18.3$ \\
\hline $\mathrm{LDL}-\mathrm{C}(\mathrm{mg} / \mathrm{dL})$ & $124.8 \pm 29.4$ & $126.2 \pm 27.7$ & $137.4 \pm 32.2$ & $120.5 \pm 30.2$ \\
\hline
\end{tabular}

The data is presented as mean \pm standard deviation.

MHT: menopausal hormone therapy, MP4: micronized progesterone, BMI: body mass index, BP: blood pressure, Total-C: total cholesterol, TG: triglycerides, HDL: high-density lipoprotein, LDL: low-density lipoprotein.

${ }^{*}, t, \neq, \$ p<0.05$ by Wilcoxon rank sum test or $t$ test.

Table 2. Blood CRP level in study subjects

\begin{tabular}{cccccr}
\hline \multirow{2}{*}{ Month } & \multicolumn{2}{c}{ Oral MHT $(n=89)$} & & \multicolumn{2}{c}{ Percutaneous MHT $(n=40)$} \\
\cline { 2 - 3 } \cline { 5 - 6 } & Estrogen only $(n=29)$ & Estrogen + MP4 $(n=60)$ & & Estrogen only* $(n=18)$ & Estrogen + MP4 $(n=22)$ \\
\hline 0 & $0.13 \pm 0.28$ & $0.08 \pm 0.08$ & $0.09 \pm 0.12$ & $0.06 \pm 0.07$ \\
3 & $0.11 \pm 0.18$ & $0.12 \pm 0.17$ & $0.06 \pm 0.05^{+}$ & $0.11 \pm 0.20$ \\
6 & $0.20 \pm 0.41$ & $0.11 \pm 0.14$ & $0.10 \pm 0.15^{+}$ & $0.08 \pm 0.07$ \\
\hline
\end{tabular}

The data is presented as mean \pm standard deviation. A logarithmic transformation of the CRP was performed due to a skewed distribution.

CRP: C-reactive protein, MHT: menopausal hormone therapy, MP4: micronized progesterone.

*There was a significant time trend $(P<0.05$ by generalized estimating equations) within the percutaneous estrogen group, after controlling for age, menopausal age, body mass index, and basal CRP; ${ }^{\dagger} P<0.05$ by generalized estimating equations after controlling for age, menopausal age, body mass index, and basal CRP; There were no statistical differences in both the oral and percutaneous MHTs according to addition of MP4.

were examined. Compared with baseline, no significant difference in CRP was observed at three or six months within the oral estrogen group $(n=29)$. The CRP level was also comparable during follow-up within the percutaneous estrogen group $(n=18)$. However, the CRP levels at three months were significantly different from those at six months $(P=$
0.0021) and there was a significant time trend $(P=0.0013)$ within the percutaneous estrogen group but not within the oral estrogen group. The difference between the two groups did not reach statistical significance.

The impacts of MP4 on estrogen therapy were investigated next (Table 2). Numbers of estrogen + MP4 in the oral and 
percutaneous MHT groups were 60 and 22, respectively. There was no difference in CRP at each time point between the estrogen alone and estrogen + MP4 treatments in both the oral and percutaneous MHT groups. The pattern of CRP change did not differ according to addition of MP4 in either MHT group.

\section{Discussion}

We report that oral estrogen therapy did not influence CRP in postmenopausal Korean women. This study also suggests that percutaneous estrogen might inhibit serum CRP level. In addition, MP4 showed no influence on the effects of estrogen.

In previous studies, CRP level was elevated with $\mathrm{CEE}$. $^{19,21,25}$ However, a CRP increase did not correlate with other inflammatory markers. Oral estrogen therapy did reduce the circulating levels of E-selectin, intercellular adhesion molecule-1, vascular cell adhesion molecule-1, monocyte chemoattractant protein-1 (MCP-1), IL-6, and TNF- $\alpha{ }^{19,20}$ Direct anti-inflammatory effects of estrogen are substantiated by in-vitro studies. Estradiol inhibits MCP-1 mRNA expression in macrophage. ${ }^{26}$ Estradiol also attenuates cell adhesion molecules in vascular endothelial cell. ${ }^{27}$ Elevation of CRP is possibly due to the first-pass hepatic effect of oral administration $^{28}$ because oral estrogen lowers IL- 6 and TNF- $\alpha$, the modulators of the liver production of CRP. Indeed, transdermal estrogen showed no change in or even sometimes lowered the CRP level. ${ }^{21,25}$ Subsequent studies have shown that this increase in CRP with oral estrogen therapy is less likely to be associated with inflammation. ${ }^{19,28}$

In this study, we tested the effects of percutaneous estrogen. Percutaneous gel is another parenteral preparation for estrogen that avoids hepatic first-pass effects. The gel passes through the skin and then forms a reservoir in the epidermal layer for continuous release of estrogen. ${ }^{29}$ Compared to patches, this gel has weaker adverse skin effects and could provide higher estradiol serum values with less day-to-day variation. $^{30}$

This study showed that follow-up CRP levels were not different from the baseline value in the percutaneous estrogen group. But three-month CPR was significantly lower than six- month CPR and there was a significant time trend for CRP within the group. These findings suggest an early decline in CRP with percutaneous estrogen, which is in line with antiinflammatory action. The results of the current study are also consistent with previous studies that have reported no change or even a decrease in CRP level with a transdermal route. ${ }^{21,25}$ Of note, oral estrogen did not change CRP in postmenopausal Korean women. Asians usually have a lower CRP level than most Westerners by as much as $1 / 10,^{31}$ and most are less than $1 \mathrm{mg} / \mathrm{L}^{32} \mathrm{CRP}$ level is also varied among East Asians and is higher in Chinese and Taiwanese people than in Koreans and Japanese. ${ }^{32}$ This variation in baseline CRP level between countries might be attributed to different CRP responses to estrogen therapy. Further prospective study is needed to clarify this possible ethnic difference.

In MHT, the addition of a progestogen to estrogen is required for endometrial protection. ${ }^{33}$ There are several options for progestogen. MPA and MP4 are equally effective for endometrial safety. ${ }^{32}$ However, MPA may counteract some of the favorable cardiovascular effects of estradiol, possibly due to its glucocorticoid activity, which is not the case for progesterone. ${ }^{34}$ In this study, MP4 was used as a progestogen because it seemed safer than MPA. ${ }^{35}$ This study suggested that oral MP4 did not affect estrogen's impacts regardless of the route of administration in postmenopausal Korean women, which is consistent with the findings of a Brazilian study that assessed vaginal MP4. ${ }^{34}$

A limitation of this study is its retrospective study design. In addition, the sample size was too small to have full statistical power. To clarify the effects of MHT on CRP in postmenopausal Korean women, a randomized controlled trial with a more appropriate sample size will be needed.

In conclusion, no change in CRP was observed with oral estrogen therapy, whereas percutaneous estrogen might decrease serum CRP level in postmenopausal Korean women, in line with its anti-inflammatory action. The estrogenic impacts on CRP were not influenced by adding MP4 in either the oral or parenteral route of estrogen administration.

\section{Acknowledgements}

This work has been supported, in part, by the Sam- 
sung Medical Center Research Fund (S-2011-0683-000 and PHO1133361) and the IN-SUNG Foundation for Medical Research (C-A5-811-1).

\section{Conflict of Interest}

No potential conflict of interest relevant to this article was reported.

\section{References}

1. Ridker PM, Hennekens CH, Buring JE, Rifai N. C-reactive protein and other markers of inflammation in the prediction of cardiovascular disease in women. N Engl J Med 2000; 342: 836-43.

2. Ridker PM, Hennekens CH, Rifai N, Buring JE, Manson JE. Hormone replacement therapy and increased plasma concentration of C-reactive protein. Circulation 1999; 100: 713-6.

3. Haverkate F, Thompson SG, Pyke SD, Gallimore JR, Pepys MB. Production of Creactive protein and risk of coronary events in stable and unstable angina. European Concerted Action on Thrombosis and Disabilities Angina Pectoris Study Group. Lancet 1997; 349: 462-6.

4. Tracy RP, Lemaitre RN, Psaty BM, Ives DG, Evans RW, Cushman M, et al. Relationship of C-reactive protein to risk of cardiovascular disease in the elderly. Results from the Cardiovascular Health Study and the Rural Health Promotion Project. Arterioscler Thromb Vasc Biol 1997; 17: 1121-7.

5. Ridker PM, Cushman M, Stampfer MJ, Tracy RP, Hennekens $\mathrm{CH}$. Inflammation, aspirin, and the risk of cardiovascular disease in apparently healthy men. N Engl J Med 1997; 336: 973-9.

6. Kuller LH, Tracy RP, Shaten J, Meilahn EN. Relation of Creactive protein and coronary heart disease in the MRFIT nested case-control study. Multiple Risk Factor Intervention Trial. Am J Epidemiol 1996; 144: 537-47.

7. Buckley DI, Fu R, Freeman M, Rogers K, Helfand M. C-reactive protein as a risk factor for coronary heart disease: a systematic review and meta-analyses for the U.S. Preventive Services Task Force. Ann Intern Med 2009; 151: 483-95.

8. Reslan OM, Khalil RA. Vascular effects of estrogenic menopausal hormone therapy. Rev Recent Clin Trials 2012; 7: 47-70.

9. Korean Statistical Information Service (KOSIS). The top 50 of the most common disease mortality rate [Internet]. Daejeon: KOSIS, 2018. [cited by 2018 April 1]. Available from: http://kosis.kr/statHtml/statHtml. do?orgId=101\&tblId=DT 1B34E13\&vw_cd=MT_ZTITLE\&list_id=D11\&seqNo=\&lang_ mode $=$ ko\&language $=$ kor\&obj_var_id $=\& i t m \_i d=\& c o n n$ path=MT_ZTITLE.

10. Yilmazer M, Fenkci V, Fenkci S, Sonmezer M, Aktepe O, Altindis $\mathrm{M}$, et al. Hormone replacement therapy, C-reactive protein, and fibrinogen in healthy postmenopausal women. Maturitas 2003; 46: 245-53.

11. Wenger NK, Speroff L, Packard B. Cardiovascular health and disease in women. N Engl J Med 1993; 329: 247-56.

12. Mosca L. The role of hormone replacement therapy in the prevention of postmenopausal heart disease. Arch Intern Med 2000; 160: 2263-72.

13. Mosca L. Hormone replacement therapy in the prevention and treatment of atherosclerosis. Curr Atheroscler Rep 2000; 2: 297-302.

14. Grodstein F, Stampfer MJ, Manson JE, Colditz GA, Willett WC, Rosner B, et al. Postmenopausal estrogen and progestin use and the risk of cardiovascular disease. N Engl $\mathrm{J}$ Med 1996; 335: 453-61.

15. Atkins CD. Postmenopausal hormone therapy and mortality. N Engl J Med 1997; 337: 1390-1.

16. Sellers TA, Mink PJ, Cerhan JR, Zheng W, Anderson KE, Kushi $\mathrm{LH}$, et al. The role of hormone replacement therapy in the risk for breast cancer and total mortality in women with a family history of breast cancer. Ann Intern Med 1997; 127: 973-80.

17. Boardman HM, Hartley L, Eisinga A, Main C, Roqué i Figuls M, Bonfill Cosp X, et al. Hormone therapy for preventing cardiovascular disease in post-menopausal women. Cochrane Database Syst Rev 2015; (3): CD002229.

18. Koh KK. Effects of estrogen on the vascular wall: vasomotor function and inflammation. Cardiovasc Res 2002; 55: 714-26.

19. Silvestri A, Gebara O, Vitale C, Wajngarten M, Leonardo F, Ramires JA, et al. Increased levels of Creactive protein after oral hormone replacement therapy may not be related to an increased inflammatory response. Circulation 2003; 107: 3165-9.

20. Koh KK, Ahn JY, Jin DK, Yoon BK, Kim HS, Kim DS, et al. Effects of continuous combined hormone replacement therapy on inflammation in hypertensive and/or overweight postmenopausal women. Arterioscler Thromb Vasc Biol 2002; 22: 1459-64.

21. Vehkavaara S, Silveira A, Hakala-Ala-Pietilä T, Virkamäki A, Hovatta O, Hamsten A, et al. Effects of oral and transdermal estrogen replacement therapy on markers of coagula- 
tion, fibrinolysis, inflammation and serum lipids and lipoproteins in postmenopausal women. Thromb Haemost 2001; 85: $619-25$.

22. Rossouw JE, Anderson GL, Prentice RL, LaCroix AZ, Kooperberg C, Stefanick ML, et al. Risks and benefits of estrogen plus progestin in healthy postmenopausal women: principal results from the women's health initiative randomized controlled trial. JAMA 2002; 288: 321-33.

23. Rosano GM, Webb CM, Chierchia S, Morgani GL, Gabraele M, Sarrel PM, et al. Natural progesterone, but not medroxyprogesterone acetate, enhances the beneficial effect of estrogen on exercise-induced myocardial ischemia in postmenopausal women. J Am Coll Cardiol 2000; 36: 2154-9.

24. Yousuf O, Mohanty BD, Martin SS, Joshi PH, Blaha MJ, Nasir K, et al. High-sensitivity C-reactive protein and cardiovascular disease: a resolute belief or an elusive link? J Am Coll Cardiol 2013; 62: 397-408.

25. Modena MG, Bursi F, Fantini G, Cagnacci A, Carbonieri A, Fortuna A, et al. Effects of hormone replacement therapy on C-reactive protein levels in healthy postmenopausal women: comparison between oral and transdermal administration of estrogen. Am J Med 2002; 113: 331-4.

26. Frazier-Jessen MR, Kovacs EJ. Estrogen modulation of JE/ monocyte chemoattractant protein-1 mRNA expression in murine macrophages. J Immunol 1995; 154: 1838-45.

27. Caulin-Glaser T, Watson CA, Pardi R, Bender JR. Effects of 17beta-estradiol on cytokine-induced endothelial cell adhesion molecule expression. J Clin Invest 1996; 98: 36-42.
28. Vongpatanasin W, Tuncel M, Wang Z, Arbique D, Mehrad B, Jialal I. Differential effects of oral versus transdermal estrogen replacement therapy on C-reactive protein in postmenopausal women. J Am Coll Cardiol 2003; 41: 1358-63.

29. Choi DS, Lee DY, Yoon BK. Effects of transdermal estrogen gel in postmenopausal Korean women. J Korean Soc Menopause 2012; 18: 113-8.

30. Scott RT Jr, Ross B, Anderson C, Archer DF. Pharmacokinetics of percutaneous estradiol: a crossover study using a gel and a transdermal system in comparison with oral micronized estradiol. Obstet Gynecol 1991; 77: 758-64.

31. Kao PC, Shiesh SC, Wu TJ. Serum Creactive protein as a marker for wellness assessment. Ann Clin Lab Sci 2006; 36: 163-9.

32. Saito I, Maruyama K, Eguchi E. C-reactive protein and cardiovascular disease in East asians: a systematic review. Clin Med Insights Cardiol 2015; 8(Suppl 3): 35-42.

33. Furness S, Roberts H, Marjoribanks J, Lethaby A. Hormone therapy in postmenopausal women and risk of endometrial hyperplasia. Cochrane Database Syst Rev 2012; (8): CD000402.

34. L'hermite M, Simoncini T, Fuller S, Genazzani AR. Could transdermal estradiol + progesterone be a safer postmenopausal HRT? A review. Maturitas 2008; 60: 185-201.

35. L'Hermite M. HRT optimization, using transdermal estradiol plus micronized progesterone, a safer HRT. Climacteric 2013; 16 Suppl 1: 44-53. 